\title{
Newborn Care Practice and Associated Factors among Mothers of One-Month-Old Infants in Southwest Ethiopia
}

\author{
Amanuel Nuramo Sakelo $\left(\mathbb{D},{ }^{1}\right.$ Nega Assefa, $^{2}$ Lemessa Oljira, $^{2}$ and Zebene Mekonnen Assefa ${ }^{3}$ \\ ${ }^{1}$ Department of Midwifery, College of Medicine and Health Science, Wachamo University, Ethiopia \\ ${ }^{2}$ Department of Public Health, College of Health and Medical Science, Haramaya University, Ethiopia \\ ${ }^{3}$ Department of Nursing, College of Medicine and Health Science, Wolkite University, Ethiopia
}

Correspondence should be addressed to Amanuel Nuramo Sakelo; legaamanuel@gmail.com

Received 5 April 2020; Revised 23 July 2020; Accepted 4 October 2020; Published 20 October 2020

Academic Editor: Francesco Porta

Copyright (C) 2020 Amanuel Nuramo Sakelom et al. This is an open access article distributed under the Creative Commons Attribution License, which permits unrestricted use, distribution, and reproduction in any medium, provided the original work is properly cited.

\begin{abstract}
Newborn care refers to the care that is provided to the baby from birth to one-month-old by a caregiver or by the mothers including thermal care, hygienic care, cord care, eye care, breastfeeding, immunization, and identification of newborn danger signs. According to Ethiopian Demographic and Health Survey (EDHS) 2016, the neonatal mortality rate was 29 deaths per 1000 live births, and the postneonatal mortality rate was 19 deaths per 1000 live births with neonates contributing 48 deaths per 1000 of the infant mortality. Neonatal mortality accounts for approximately two-thirds of all infant mortality worldwide. Objective. The objective of this study was to assess newborn care practice and associated factors among mothers with babies of one-month-old in Hossana town, Southern Nations, Nationalities, and Peoples' Region, Ethiopia, 2018. Methods. A community-based crosssectional study was conducted among randomly selected 422 mothers with babies of one-month-old in Hossana town, southwest Ethiopia. The data were entered to EpiData 3.1 and exported to Statistical Package for the Social Sciences (SPSS) version 22. Bivariate and multivariate analyses were applied, and frequencies and odds ratios were calculated to determine the prevalence and associated factors, respectively. Results. In this study, 31\% of participants had good newborn care practice based on three composite variables such as $84 \%$ who have done early breastfeeding initiation, $32.9 \%$ who have done safe cord care, and $30.6 \%$ who have done thermal care. Educational status of the mother's, primary (AOR $=2.80,95 \% \mathrm{CI}: 1.027-7.637$ ), secondary $(\mathrm{AOR}=2.596,95 \% \mathrm{CI}: 0.921-7.316)$, and college and above (AOR $=3.63,95 \% \mathrm{CI}$ : 1.056-12.492); mothers who practiced handwashing (hygiene) before touching a newborn (AOR =2.552, 95\% CI: 1.092-5.963); and mothers who had good knowledge on newborn care practice ( $\mathrm{AOR}=15.638,95 \% \mathrm{CI}$ : $3.599-67.943$ ) were significantly associated with newborn care practice. Conclusion and Recommendation. The present study indicated that the level of comprehensive newborn care practice was unsatisfactory; all responsible bodies were giving attention and intervene on the predictors to improve newborn care practice and provide health education regarding newborn care practice. Education level, health education (counseling) on hygiene, and knowledge of mother on newborn care practice were independent predictors of newborn care practice.
\end{abstract}

\section{Introduction}

Essential newborn care is the basic care required for every baby and comprises thermal care (delayed bathing, drying, and keeping the baby warm through skin-to-skin contact), infection prevention (promoting and supporting handwashing for all caregivers and providing hygienic umbilical cord and skin care), feeding support (early and exclusive breastfeeding), and postnatal care, including monitoring of newborns for danger signs of serious infections and identifying babies requiring additional care [1]. Deaths in the newborn period (first 28 days) are a growing proportion of all child deaths [2], and essential newborn care practice is used to decrease neonatal morbidity and mortalities if given appropriately [3].

There is a global underfive mortality rate of 42.5 per 1000 live births; of those deaths, $45 \%$ were newborns, with a 
neonatal mortality rate of 19 per 1000 live births [4, 5], and although underfive and infant mortalities have been reduced, neonatal mortality remains largely unchanged in Nepal [6].

In Ethiopia, neonatal morbidity and mortality rates were among the highest in the world [7] and neonatal mortality was found to be 214 out of 4888 live births with the rate of 43.8 per 1000 live births in north Gonder [8], and also, according to the EDHS report, neonatal mortality was 29 per 1000, 41per 1000, and 38 per 1000 in urban and rural, respectively, in Ethiopia [9].

Even only $26 \%$ of birth occur in a health facility, there is an increase in neonatal death [9]. To reduce newborn death, newborn care becomes the health priority [5]. Families, are focus on immediate newborn care at home and changes in household level practices to prevent newborn death, illness and to promote health of newborn care in Ethiopia [1]. Merely $13 \%$ of newborns receive a postnatal check within two days of birth [9]. The level of newborn care practice is scanty, inconclusive, and there are limited studies conducted in this area that focus on practices of newborn care and associated factors among mothers in this region (SNNPR).

\section{General Objective}

The objective of this study was to assess newborn care practice and associated factors among mothers of one-monthold infants in Hossana town, Hadiya zone, southern Ethiopia, 2018.

\section{Specific Objectives}

(i) To assess newborn care practice among mothers with babies aged one month

(ii) To identify factors associated with newborn care practice among mothers with babies aged one month

\section{Methods and Materials}

4.1. Study Area and Period. Hossana town is the capital city of Hadiya zone, Southern Nations, Nationalities, and Peoples' Region (SNNPR), which was located $194 \mathrm{~km}$ from Hawassa, the capital city of the region, and $230 \mathrm{~km}$ from Addis Ababa, the capital city of the country.

Hossana town is a purely woinedega agroeconomic zone, situated at an altitude of 1800-2950 meters above sea level, and has an average temperature ranging from 10 to 24 degree centigrade. The annual rainfall is $1250 \mathrm{~mm}$ per year.

Based on the 2007 Ethiopian national population and housing census, the population of the town was 78,432: male 38,800 and female 39,632 ; the number of childbearing age women (15-49 years) was 18,275 (Hossana town administrative office report 2007).

A community-based cross-sectional study was conducted from January 20 to February 19, 2018, in Hossana town, southwest Ethiopia.

4.1.1. Study Design. A community-based cross-sectional study design was conducted.
4.1.2. Source of Population. The source population is all women in the reproductive age group, who had one-month age infants in Hossana town.

4.1.3. Study Population. Study populations were all sampled mothers who had one-month age infants during the data collection period in Hossana town.

\subsection{Inclusion and Exclusion Criteria}

4.2.1. Inclusion Criteria. Mothers resident in the area for six or more months before this study was conducted were included in the study.

4.2.2. Exclusion Criteria. Mothers who were unable to feed breast milk and too sick or critically ill during the data collection period were excluded in the study.

4.3. Sample Size Determination. The sample size was determined by using a single population proportion formula:

$$
n=\frac{(Z a / 2)^{2} p(1-p)}{D^{2}}
$$

where $n$ is the minimum sample, $p$ is the $52.1 \%$ prevalence level for early breastfeeding on four regions of Ethiopia [10], $D$ is the margin error $(0.05)$, and $Z(a / 2)$ is the standard normal.

$$
n=\frac{(1.96)^{2} \times 0.521(1-0.521)}{(0.05)^{2}}=384
$$

Then, to get the final sample size $(N)$, the nonresponse rate $(10 \%)$ was used, $384 \times 0.1=38.4+384=422$.

4.4. Sampling Procedure. A survey was conducted among 422 women having a one-month infant in Hossana town. A multistage sampling technique was used to select study participants, and the study was conducted in all kebeles found in the town. A proportional allocation to the size of the population was done to decide the number of women required from each kebeles.

Finally, a simple random sampling technique was applied to identify women to be included in the survey. When more than one eligible respondent is present in a selected household, one respondent is selected on the spot by a lottery method.

\section{Variables}

\subsection{Dependent Variable}

(i) Newborn care practice (early breastfeeding, thermal care, and cord care)

\subsection{Independent Variable}

(i) Sociodemographic and socioeconomic factors (parity, occupation, education status, and place of delivery) 
(ii) Mothers' knowledge on newborn care practice (knowledge on newborn care practice and knowledge on newborn danger signs)

(iii) Health service and obstetric factors (attendance of ANC, health education during ANC and PNC, health extension worker home visit, and neonatal death)

\subsection{Data Collection Procedures (Instruments, Personnel, and Data Quality Control)}

5.3.1. Data Collection Instruments. The data were collected using a structured interviewer-administered questionnaire adapted from the EDHS, and other relevant literatures were used to collect data. The questionnaire had included all the questions that assess the knowledge and practice of newborn care of mothers. The tool was prepared in English version and translated to Hadiyisa (local language) and then translated back to English language to check for consistency. Finally, the data were collected by Hadiyisa language.

5.3.2. Data Collection Process. Seven diploma midwives and five diploma nurses were recruited from other kebeles. Training was given for both data collectors and supervisors for two days before the actual data collection about data collection techniques go through the questionnaire questions with questions, ways of data collection, supervision and final clarification was given to those who have doubts.

5.4. Operational Definitions and Definition of Terms. Newborn care: it refers to the care provided to the baby from birth to 28 days of age by a caregiver or by the mothers including thermal care, hygienic care, cord care, eye care, breastfeeding, immunization, and identification of newborn danger signs.

Practice of newborn care: a mother was asked questions that cover the practice of newborn care which includes early initiation of breastfeeding and providing colostrums, cord care, and thermal care. The investigator developed composite index questions in the above issues that assigned a score of one [1] = correct response (consistent with the WHO essential newborn care guidelines) and $0=$ incorrect response (inconsistent with the WHO/Unicef essential newborn care guidelines); any mother who did not know the answer is considered to have an incorrect response.

Good knowledge of mothers on newborn care: those mothers who respond correctly above $50 \%$ of knowledgerelated questions.

Poor knowledge of mothers on newborn care: those mothers who respond correctly less than or equal to $50 \%$ of knowledge-related questions.

Newborn care practice: good newborn care practice: those mothers who mentioned three newborn care practices; poor newborn care practice: those who reported two or less newborn care practices.

Kebele: it is the smallest administrative unit, similar to a ward, a neighborhood, or a localized and delimited group of people and a part of woreda (district).
5.5. Data Quality Management. Data quality was assured by using a pretested data collection tool, and training was given for data collectors and supervisors before actual data collection. Supervisors were engaged in continuous supervision and monitoring during data collection. Completeness and consistency of data were checked by supervisors, data clerks, and investigators before and during data entry.

5.6. Data Analysis. Collected data was checked for its completeness and then coded and entered into EpiData version 3.1, and entered data was exported to SPSS version 20 for analysis. Binary and multivariate logistic regressions were employed. Frequencies and proportions were computed. A significant association was determined by odds ratios with $p$ value $<0.05$, at $95 \%$ confidence interval. Finally, the results were presented in the form of tables, figures, and charts as appropriate.

\section{Results}

A total of 422 mothers who had one-month infants were involved in the study, yielding a $100 \%$ response rate. The majority of respondents, 289 (68.5\%), were between 25 and 34 years of age, 213 (50.5\%) were housewives, 418 (99.1\%) were married, $258(61.1 \%)$ were protestant religion, 279 (66.1\%) were Hadiya ethnicity, $378(89.6 \%)$ had a formal education, and $46(10.9 \%)$ had a high income (Table 1$)$.

6.1. Health Care Service Utilization and Obstetric Information. From the participants, 303 (71.8\%) had a home visit in the last one month and had health educations; 369 (87.4\%) had knowledge on handwashing with soap and clean water before handling their neonate, $347(82.2 \%)$ on keeping neonate dry and wrapping after delivery, 402 $(95.3 \%)$ on breastfeeding immediately after birth within an hour, $383(90.8 \%)$ on danger sign, $373(88.4 \%)$ on immunization, and $361(85.5 \%)$ on how to care for low-birth-weight baby by HEW.

The majority of participants, 351 (83.2\%), had no history of neonatal death before this delivery, 411 (97.4\%) had ANC follow-up when they were pregnant, and 405 (96.0\%) had given birth at health facilities (Table 2).

6.2. Initiation of Breastfeeding, Cord Care, and Thermal Care Practice. From the total participants, 409 (96.9\%) gave the first breast milk for their baby, 352 (85.4\%) initiated breast milk within an hour after birth, 336 (79.6\%) did not apply anything on the cord of the newborn baby, 214 (50.7\%) took care of bleeding to keep the cord clean and safe, 144 (34.1\%) kept the cord dry and clean to keep the cord clean and safe, and $64(15.2 \%)$ took the newborn baby to a health facility in order to keep umbilical cord clean and safe.

More than half of the respondents, 215 (50.9\%), had given the first bath for newborn baby within the first 24 hours of delivery, and 293 (69.4\%) placed the newborn baby to skinto-skin contact always until the baby becomes stable (Table 3 ).

6.3. Mothers' Knowledge on Newborn Care and Danger Signs. Among the 422 study participants, 406 (96.2\%) had known about care for their newborn, 331 (78.4\%) applied nothing 
TABLE 1: Sociodemographic variables of mothers with babies of onemonth-old in Hossana town, southwest Ethiopia, $2018(n=422)$.

\begin{tabular}{|c|c|c|}
\hline Variables $(n=422)$ & Frequency $(n)$ & Percent (\%) \\
\hline \multicolumn{3}{|l|}{ Age (year) } \\
\hline $15-24$ & 76 & 18.0 \\
\hline $25-34$ & 289 & 68.5 \\
\hline$\geq 35$ & 57 & 13.5 \\
\hline \multicolumn{3}{|l|}{ Religion } \\
\hline Protestant & 258 & 61.1 \\
\hline Orthodox & 106 & 25.1 \\
\hline Other & 58 & 13.7 \\
\hline \multicolumn{3}{|l|}{ Ethnicity } \\
\hline Hadiya & 279 & 66.1 \\
\hline Kembata & 60 & 14.2 \\
\hline Other & 83 & 19.7 \\
\hline \multicolumn{3}{|l|}{ Marital status } \\
\hline Married & 418 & 99.1 \\
\hline Others & 4 & .9 \\
\hline \multicolumn{3}{|l|}{ Educational level } \\
\hline Primary & 137 & 32.5 \\
\hline College and above & 129 & 30.6 \\
\hline Secondary & 112 & 26.5 \\
\hline No formal education & 44 & 10.4 \\
\hline \multicolumn{3}{|l|}{ Occupation } \\
\hline Housewife & 213 & 50.5 \\
\hline Governmental employee & 117 & 27.7 \\
\hline Others & 92 & 21.8 \\
\hline \multicolumn{3}{|l|}{ Wealth index } \\
\hline Lowest & 261 & 61.8 \\
\hline Middle & 115 & 27.3 \\
\hline Highest & 46 & 10.9 \\
\hline
\end{tabular}

to the cord immediately after cutting up to 7 days except ordered medication, 294 (69.7\%) handled umbilical cord after cutting without dressing, $327(77.5 \%)$ bathed her newborn baby after 24 hours after delivery, $372(88.2 \%)$ breastfed their baby within 1 hour after delivery, $368(87.2 \%)$ believed feeding breast milk as the first food for a newborn baby after delivery, and 367 (87.0\%) had knowledge about newborn danger sign (Table 4$)$.

From the total of 422 participants, the mentioned danger signs of a newborn baby are as follows: $84.8 \%$ were poor sucking, $77 \%$ were fast breathing, $64 \%$ had hypothermia, $64.7 \%$ had fever, $46 \%$ had drowsiness (unconscious), and $66.1 \%$ had cord bleeding and infection (Figure 1).

In this study, the proportion of newborn care practices was $130(30.8 \%)$ of the respondents in terms of the three composite practices, namely, 354 (83.9\%) were early breastfeeding initiation, 139 (32.9\%) were safe cord care, and 129 $(30.6 \%)$ were thermal care (delay bathing) (Figure 2).

6.4. Factors That Associated with Newborn Care Practice. In this study, education of the mothers, mothers who had
TABLE 2: Health care service utilization and obstetrics information of mothers with babies of one-month-old in Hossana town, southwest Ethiopia, $2018(n=422)$.

\begin{tabular}{|c|c|c|}
\hline Variables $(n=422)$ & $\begin{array}{l}\text { Frequency } \\
(n)\end{array}$ & $\begin{array}{c}\text { Percent } \\
(\%)\end{array}$ \\
\hline \multicolumn{3}{|l|}{ HEW home visit } \\
\hline Yes & 303 & 71.8 \\
\hline No & 119 & 28.2 \\
\hline \multicolumn{3}{|l|}{ Health education } \\
\hline Yes & 369 & 87.4 \\
\hline No & 53 & 12.6 \\
\hline \multicolumn{3}{|l|}{$\begin{array}{l}\text { Keeping immediately dry and } \\
\text { wrapping }\end{array}$} \\
\hline Yes & 347 & 82.2 \\
\hline No & 75 & 17.8 \\
\hline \multicolumn{3}{|l|}{ BF immediately within an hour } \\
\hline Yes & 402 & 95.3 \\
\hline No & 20 & 4.7 \\
\hline \multicolumn{3}{|l|}{ HEW advice on danger signs } \\
\hline Yes & 383 & 90.8 \\
\hline No & 39 & 9.2 \\
\hline \multicolumn{3}{|l|}{ HEW advice on immunization } \\
\hline Yes & 373 & 88.4 \\
\hline No & 49 & 11.6 \\
\hline \multicolumn{3}{|l|}{ HEW advice on LBW care } \\
\hline Yes & 361 & 85.5 \\
\hline No & 61 & 14.5 \\
\hline \multicolumn{3}{|l|}{ History of neonatal death } \\
\hline Yes & 71 & 16.8 \\
\hline No & 351 & 83.2 \\
\hline \multicolumn{3}{|l|}{ ANC follow-up } \\
\hline Yes & 411 & 97.4 \\
\hline No & 11 & 2.6 \\
\hline \multicolumn{3}{|l|}{ Place of current ANC visit } \\
\hline Governmental health facility & 376 & 89.1 \\
\hline Private health facility & 46 & 10.9 \\
\hline \multicolumn{3}{|l|}{ Place of current delivery } \\
\hline Home & 17 & 4.0 \\
\hline Health facility & 405 & 96.0 \\
\hline
\end{tabular}

HEW = health extension workers; LBW = low birth weight; $\mathrm{ANC}=$ antenatal care.

practice handwashing, and knowledge of mothers on newborn care practice were significantly associated with newborn care practice.

Hence, those who had primary, secondary, and college and above educational status had three times $(\mathrm{AOR}=2.80$, 95\% CI: $1.027-7.637)$, three times $(\mathrm{AOR}=2.596,95 \% \mathrm{CI}$ : $0.921-7.316$ ), and four times (AOR $=3.63$, 95\% CI: 1.056 12.492) more likely to practice newborn care than mothers who had no formal education, respectively, mothers who had practice handwashing were three times more likely to practice newborn care than mothers who had not practice 
TABle 3: Practice of initiation of breastfeeding, cord care, and thermal care of mothers with babies of one-month-old in Hossana town, southwest Ethiopia, $2018(n=422)$.

\begin{tabular}{lcc}
\hline Variables $(n=422)$ & $\begin{array}{c}\text { Frequency } \\
(n)\end{array}$ & $\begin{array}{c}\text { Percent } \\
(\%)\end{array}$ \\
\hline Did you give/feed colostrum & 409 & 96.9 \\
$\quad$ Yes & 13 & 3.1 \\
$\quad$ No & 352 & 85.4 \\
When did you initiate breast milk & 70 & 14.6 \\
$\quad$ Within an hour & & \\
After one hour & 86 & 20.4 \\
Did you apply anything on the cord & 336 & 79.6 \\
$\quad$ Yes & & \\
$\quad$ No & 214 & 50.7 \\
What did you do to keep the cord safe & 144 & 34.1 \\
Taking care of bleeding & 64 & 15.2 \\
Keeping it dry and clean & & \\
Taking to a health facility & 215 & 50.9 \\
When did you give a bath to baby & 207 & 49.1 \\
Within 24 hours & & \\
After 24 hours & 129 & 30.6 \\
Was placed in skin-to-skin 1st & 293 & 69.4 \\
Not at all & & \\
Always & & \\
\hline
\end{tabular}

TABLE 4: Knowledge on newborn care and danger signs of mothers with babies of one-month-old in Hossana town, southwest Ethiopia, $2018(n=422)$.

\begin{tabular}{lcc}
\hline Variables $(n=422)$ & Frequency $(n)$ & Percent $(\%)$ \\
\hline Mother care to newborn baby & 406 & 96.2 \\
Yes & 16 & 3.8 \\
No & 331 & \\
What substance was applied to the cord & 78.4 \\
Nothing applied & 91 & 21.6 \\
Butter applied & 128 & \\
How long was the cord handled after cutting & 30.3 \\
With dressing/cover & 294 & 69.7 \\
Without dressing & 95 & 22.5 \\
How long after birth was the baby washed for the $1^{\text {st }}$ time \\
Within 24 hours & 327 & 77.5 \\
After 24 hours & 372 & \\
How long after birth should the baby be breastfed & \\
Within one hour & 50 & 11.8 \\
After one hour & & \\
What should mother feed baby first & 368 & 13.0 \\
Breast milk & 54 & \\
Other than breast milk & 367 & \\
Newborn danger sign & 55 & \\
Yes & & \\
No & & \\
\hline
\end{tabular}

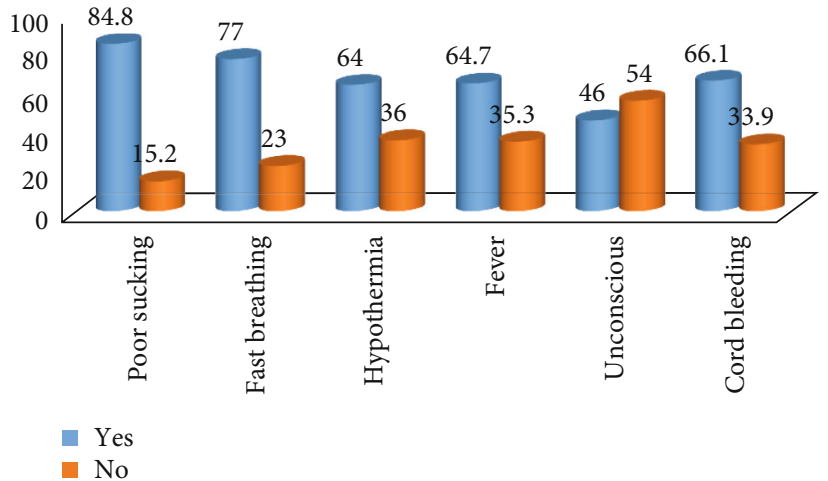

FIGURE 1: Identified newborn danger sign by mothers with babies of one-month-old after delivery in the community of Hossana town, southwest Ethiopia, 2018.

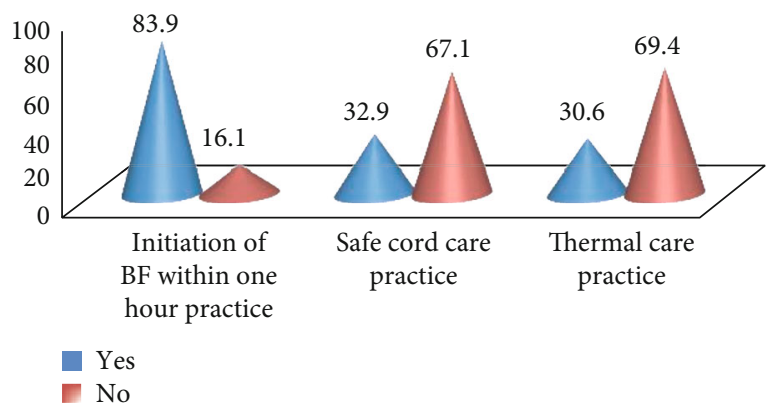

FIgURE 2: Identified three composite newborn care practices separately by mothers with babies of one-month-old after delivery in the community of Hossana town, southwest Ethiopia, 2018.

handwashing ( $\mathrm{AOR}=2.552,95 \% \mathrm{CI}: 1.092-5.963)$, and mothers who had good knowledge on newborn care practice were sixteen times more likely to practice newborn care than those who had poor knowledge $(\mathrm{AOR}=15.638,95 \% \mathrm{CI}$ : 3.599-67.943) (Table 5).

\section{Discussion}

In this study, one-third of the participants had good newborn care practice based on three composite variables such as early breastfeeding initiation $83.9 \%$, safe cord care $32.9 \%$, and thermal care $30.6 \%$.

Good newborn care practice was almost nearly similar to study done in Aksum Town, North Ethiopia (26.7\%) [11], but this study was lower than the study done in Mandura District, Northwest Ethiopia (40.6\%), Gulomekada District, Eastern Tigray (92.9\%), Mekelle City, North Ethiopia $(81.1 \%)[7,12,13]$, and Damot pulasa Woreda, southern Ethiopia (24\%) [14], and the difference may be due to socioeconomic, access of awareness among the study participants, geographical variation, and health-seeking behavior across the different cultures or cultural beliefs.

Breastfeeding $83.9 \%$ which was higher than study done in Hoima District, western Uganda 31\% dry cord care $60.5 \%$ [15], Mandura District, Northwest Ethiopia (48.1\%) [7], tamale metropolis of Ghana 70.5\% [16], Tharu, Chitwan 
TABLE 5: Factors that are associated with newborn care practice of mothers with babies of one-month-old in Hossana town, southwest Ethiopia, 2018.

\begin{tabular}{|c|c|c|c|c|}
\hline Variables $(n=422)$ & Good practice of NBC & Poor practice of $\mathrm{NBC}$ & AORs $(95 \% \mathrm{CI})$ & $p$ value \\
\hline \multicolumn{5}{|l|}{ Education level } \\
\hline Primary & $46(33.6 \%)$ & $91(66.4 \%)$ & $2.801(1.027-7.637)$ & $0.044^{*}$ \\
\hline Secondary & $33(29.5 \%)$ & $79(70.5 \%)$ & $2.596(0.921-7.316)$ & 0.071 \\
\hline College and above & $46(35.7 \%)$ & $83(64.3 \%)$ & $3.633(1.056-12.492)$ & $0.041^{*}$ \\
\hline No education & $6(13.6 \%)$ & $38(86.4 \%)$ & 1 & 1 \\
\hline \multicolumn{5}{|c|}{ Health education (hand wash) } \\
\hline Yes & $123(33.3 \%)$ & $246(66.7 \%)$ & $2.552(1.092-5.963)$ & $0.030^{*}$ \\
\hline No & $8(15.1 \%)$ & $45(84.9 \%)$ & 1 & 1 \\
\hline \multicolumn{5}{|c|}{ Mothers' knowledge on newborn care practice } \\
\hline Good & $129(35.4 \%)$ & $235(64.6 \%)$ & $15.638(3.599-67.943)$ & $<0.001^{*}$ \\
\hline Poor & $2(3.4 \%)$ & $56(96.6 \%)$ & 1 & 1 \\
\hline
\end{tabular}

*Significant association.

district 52.5\% [6] and lower than study done in Mekelle City, North Ethiopia 97.4\% [13], Aksum Town, North Ethiopia $63.1 \%[11]$.

Safe cord care was $32.9 \%$ and similar to the study done in Hoima District, western Uganda (31\%) [15], but lower than the study done in Tharu, Chitwan district (95\%) [6], Aksum Town, North Ethiopia (42.8\%) [11], and Mewat, Haryana, India (49\%) [17].

Thermal care was $30.6 \%$ in this study and nearly similar to the study done in Aksum Town, North Ethiopia (32.6\%) [11], but lower than the study done in Hoima District, western Uganda (67.2\%) [15], Mandura District, Northwest Ethiopia (37.8\%) [7], Mekelle City, North Ethiopia (66.9\%) [13], and Tharu, Chitwan district (96.6\%) [6], and this difference may be due to relatively an increased awareness about the harmful effect of traditional foreign substance application to the umbilical cord.

Knowledge of the mother on newborn care practice had a significant association with newborn care practice and similar to the study in Hoima District, western Uganda, Gulomekada District, Eastern Tigray, and Mekelle City, North Ethiopia [12, 13, 15].

Education of the mothers (primary, secondary, and college and above) also has a significant association with newborn care practice and was similar to the study done in Mandura District, Northwest Ethiopia, and Mekelle City, North Ethiopia [7, 13].

Mothers who had health education on hygiene (hand) had a significant association with newborn care practice in this study, but no study was similar to this result.

\section{Conclusions}

In this study, almost one-third of the mothers had good newborn care practice and it was very low when compared with other studies done in the country. Mothers' educational status, mothers who had health education on hygiene, and knowledge of mothers on newborn care practice were independent predictors of newborn care practice.

\section{Recommendations}

Based on the findings of this study, we recommend the following:

(i) Hadiya Zone Health Bureau: to work hard on the promotion of health facility delivery system and to increase the level of newborn care practice and involve health extension workers to apply hometo-home visit program to convince all childbearing women on the positive outcome of health facility delivery service to have good newborn care practice

(ii) Health care providers: to provide ongoing education and counseling to mothers to give birth at a health facility to have good newborn care practice during ANC follow-up

(iii) Health care planners: to provide health education during ANC and PNC regarding these predictors

(iv) Future researcher: we suggest researchers to undertake repeatable studies in this area, and as this study lacks qualitative information that can underpin the quantitative study results, we recommend that the researcher have to do qualitative study design and other methods

\section{Limitation of the Study}

The cross-sectional nature of the study is impossible to establish a temporal relationship between newborn care practice and identified risk factors.

\section{Abbreviations}

ANC: Antenatal care

CSA: Central Statistical Agency

EDHS: Ethiopian Demographic and Health Survey

ENBC: Essential newborn care

HEW: Health extension worker

ICF: Infant and children feeding 
IHRERC: Institutional Health Research Ethics Review Committee

PNC: Postnatal care

USAID: United States Agency for International Development

VDCs: Village Development Communities.

\section{Data Availability}

The authors confirm that the data supporting the findings of this study are available within the article and its supplementary materials.

\section{Ethical Approval}

Ethical clearance letters were obtained from the Institutional Health Research and Ethics Review Committee (IHRERC) of Haramaya University College of Health and Medical Science, and the letter was submitted to the Hadiya zone for permission.

\section{Consent}

Participants were informed clearly, and their consent was obtained, their confidentiality was maintained by giving code, personal privacy and cultural norms were respected, and the respondent had the right not to participate in the study or withdraw from the study at any time.

\section{Conflicts of Interest}

The authors declare that there is no competing interest.

\section{Authors' Contributions}

The authors' contribution to this study is conducting and preparing the manuscript.

\section{Acknowledgments}

First of all, we would like to thank Haramaya University College of Health Science and Medicine for providing this opportunity. Then, we would like to thank our study participants, data collectors, and supervisors.

\section{References}

[1] Unicef and Save the Children, Newborn health in humanitarian settings, United Nation, 2015.

[2] World Health Statistics, Monitoring health for the Sustainable Development Goals (SDGs), World Health Organization, Geneva, 2013.

[3] B. Darling, R. S. Wankhede, and B. A. Benjamin, "Newborn care among postnatal mothers inselected maternity centersin Madurai , Tamilnadu," International Journal of Allied Medical Sciences and Clinical Research, vol. 2, no. 2, pp. 119-124, 2014.

[4] World Health Statistics, Monitoring health for the Sustainable Development Goals (SDGs), World Health Organization, Geneva, 2017.
[5] World Health Statistics, Monitoring health for the Sustainable Development Goals (SDGs), World Health Organization, Geneva, 2016.

[6] J. Chaudhary, G. P. Dhungana, and H. C. Ghimire, "Factors affecting newborn care practices among Tharu mothers in selected Vilalge development committees of Chitwan Distract," Journal of Chitwan Medical College, vol. 3, no. 1, pp. 42-45, 2013.

[7] T. Tegene, A. N. Gashaw Andargi, and KY, "Newborn care practice and associated factors among mothers who gave birth within one year in Mandura District, northwest Ethiopia," Clinics Mother Child Health, vol. 12, no. 1, pp. 1-7, 2015.

[8] B. Kebede, A. Gebeyehu, H. R. Sharma, and S. Yifru, "Prevalence and associated factors of neonatal mortality in North Gondar Zone, northwest Ethiopia," Ethiopian Journal of Health Development, vol. 26, no. 2, pp. 66-71, 2012.

[9] Central Statistical Agency (CSA) [Ethiopia] and ICF, Ethiopia Demographic and Health Survey, Central Statistical Agency (CSA) [Ethiopia] and ICF, Ethiopia, 2016.

[10] J. A. Callaghan-Koru, A. Seifu, M. Tholandi et al., "Newborn care practices at home and in health facilities in 4 regions of Ethiopia," BMC Pediatrics, vol. 13, no. 1, pp. 1-11, 2013.

[11] M. Berhe, A. A. Medhaniye, G. Kahsay, E. Birhane, and M. Abay, "Essential neonatal care utilization and associated factors among mothers in public health facilities of Aksum town, north Ethiopia, 2016," PLOS One, vol. 12, no. 4, pp. e0175902-e0175911, 2017.

[12] H. G. Misgna, H. B. Gebru, and M. M. Birhanu, "Knowledge, practice and associated factors of essential newborn care at home among mothers in Gulomekada District, Eastern Tigray, Ethiopia, 2014," BMC Pregnancy and Childbirth, vol. 16, no. 1, p. 144, 2016.

[13] T. A. Berhea, A. B. Belachew, and G. F. Abreha, "Knowledge and practice of essential newborn care among postnatal mothers in Mekelle City, north Ethiopia : a population-based survey," PLoS One, vol. 13, no. 8, pp. 1-12, 2018.

[14] T. Y. Chichiabellu, B. Mekonnen, F. H. Astawesegn, B. W. Demissie, and A. A. Anjulo, "Essential newborn care practices and associated factors among home delivered mothers in Damot pulasa Woreda, southern Ethiopia," Reproductive Health, vol. 15, no. 1, p. 162, 2018.

[15] L. Kabwijamu, P. Waiswa, V. Kawooya, C. K. Nalwadda, M. Okuga, and E. L. Nabiwemba, "Newborn care practices among adolescent mothers in Hoima District, western Uganda," PLOS ONE, vol. 11, no. 11, pp. e0166405e0166412, 2016.

[16] R. N. Nukpezah, S. V. Nuvor, and J. Ninnoni, "Knowledge and practice of exclusive breastfeeding among mothers in the tamale metropolis of Ghana," Reproductive Health, vol. 15, no. 1, pp. 140-149, 2018.

[17] L. N. Sinha, P. Kaur, R. Gupta, S. Dalpath, V. Goyal, and M. Murhekar, "Newborn care practices and home-based postnatal newborn care programme - Mewat, Haryana, India, 2013," Western Pacific Surveillance and Response, vol. 5, no. 3, pp. 22-29, 2014. 